\title{
Modelado probabilístico de los sismos que afectan a la ciudad de Cumaná, Venezuela
}

\author{
| Probabilistic modeling of the earthquakes that affect the city of Cumaná, Venezuela |
}

\author{
Andrés A. Velásquez Gutiérrez \\ anve6894@gmail.com \\ Liceo Humberto Fernández M. \\ Ministerio de Educación - Venezuela
}

\begin{abstract}
Resumen: En esta investigación, partiendo de un conjunto de datos sobre sismos que han impactado la ciudad de Cumaná, Venezuela, se realiza un modelaje probabilístico para determinar las zonas sismogénicas que afectan a esta urbe. Se utiliza la estadística de valores extremos para estudiar series de datos que exceden cierto umbral. Estas series están contenidas en un catálogo tomado de una investigación previa derivado de los registros instrumentales del Centro de Sismología de la Universidad de Oriente, de la Fundación Venezolana de Investigaciones Científicas (FUNVISIS), del National Earthquake Information Center (NEIC) y del United States Geological Survey (USGS). Se dividieron los datos según su profundidad focal; luego utilizando la densidad sísmica, la geología y la ocurrencia de sismos históricos se identificaron las zonas sismogénicas y utilizando la ley de recurrencia de Gutenberg-Ritcher se estiman los parámetros necesarios para obtener las funciones de densidad y de distribución de probabilidad asociadas.
\end{abstract}

Palabras Clave: sismos, probabilidad, umbral, excedencia, zonas sismogénicas

\begin{abstract}
In this research, based on a set of data on earthquakes that have impacted the city of Cumaná, Venezuela, a probabilistic modeling is carried out to determine the seismogenic zones that affect this city. Extreme value statistics are used to study data series that exceed a certain threshold. These series are contained in a catalog taken from a previous research derived from the instrumental records of the Seismology Center of the University of Oriente, the Venezuelan Foundation for Scientific Research (FUNVISIS), the National Earthquake Information Center (NEIC) and the United States Geological Survey (USGS). The data were divided according to their focal depth; then, using the seismic density, the geology and the occurrence of historical earthquakes, the seismogenic zones were identified and using the Gutenberg-Ritcher recurrence law, the parameters necessary to obtain the associated density and probability distribution functions were estimated.
\end{abstract}

Keywords: earthquakes, probability, threshold, exceedance, seismogenic zones 


\section{Introducción}

La Oficina Coordinadora de las Naciones Unidas para el Socorro en casos de Desastres (Undro por sus siglas en inglés) define la peligrosidad sísmica como

$$
\mathrm{H}(\text { Hazard })=\mathrm{P}\left[\mathrm{x}(\mathrm{s}) \geq \mathrm{x}_{0} ; \mathrm{t}\right]
$$

donde $\mathrm{H}$ representa la probabilidad de superación de un umbral $\mathrm{x}_{0}$ para la intensidad del movimiento $x$ en un emplazamiento s durante un tiempo t. (Undro, 1980, citado por Benito y Jiménez, 1999, pág. 14). Se entiende por intensidad del movimiento el grado de fuerza con que se realiza el movimiento y por emplazamiento se refiere el área o lugar definido donde ocurre dicho movimiento. No debe confundirse la intensidad del movimiento con la intensidad de un sismo, la cual se refiere al grado de destrucción que causan los sismos en el área afectada. En esta investigación privilegiaremos el uso de la magnitud, para efectos de la medida del tamaño de un sismo, entendida esta como la cantidad de la energía liberada por un sismo.

La ecuación (1) lleva al conjunto $X=\left\{x_{\mathfrak{i}}(s), i=1,2, \ldots\right\}$ cuyos elementos son el subconjunto de sismos cuya magnitud supera el umbral $x_{0}$. La escogencia de $x_{0}$ como umbral puede obtenerse mediante un procedimiento matemático, pero usualmente su elección es subjetiva y depende de las observaciones realizadas sobre la destructividad de los sismos que rebasan cierta magnitud, lo que presupone extrapolar que en el futuro sismos similares tendrán efectos catastróficos sobre la población.

Mientras más alto sea el umbral $x_{0}$ elegido, más pequeña será la secuencia de sismos $\left\{x_{i}(s)\right\}$ que lo superen. Esto conlleva a enmarcar este problema dentro del análisis de la cola de la distribución. (Serra, 2013).

Para esta investigación, primero se define el conjunto universal el cual son los sismos ocurridos en el área alrededor de la ciudad cuya actividad sísmica puede afectar a las estructuras que se encuentran en ésta. Esta área abarca $300 \mathrm{Km}$. alrededor de la ciudad. Este criterio deriva de la normativa seguida por el Consejo de Seguridad Nuclear (CSN) de España y la Organización Internacional de Energía Atómica (OIEA) para el estudio probabilístico de ocurrencia de terremotos con impacto en los emplazamientos de las centrales nucleares, criterio que también es utilizado para el diseño de obras ingenieriles críticas (Mayordomo, 2013). Se procede, luego, a recopilar la información existente sobre los sismos con epicentros en esta zona. A tal fin se utiliza un catálogo realizado previo a este estudio diseñado para la investigación de (Rivera, 2017). Con el fin de obtener una sola escala de trabajo, en estos datos se homogenizan los sismos a una magnitud de referencia, en este caso se utiliza la magnitud Mw. Se descartan los premonitores y las réplicas y se extraen finalmente el subconjunto de sismos que superan en magnitud el umbral $x_{0}$ elegido. En el subconjunto obtenido, se listan además los parámetros de localización, profundidad focal o profundidad de ocurrencia del sismo, frecuencia y tiempo de ocurrencia.

Estos datos se ubican en una malla longitud-latitud para visualizar la densidad sísmica, entendida esta como la cantidad de sismos presentes en un área determinada durante el período de tiempo que comprende el catálogo utilizado y constatar la ubicación de los sismos históricos y con la ayuda de un mapa geológico de la zona se procede a delinear las zonas sismogénicas. De las excedencias de este subconjunto final de sismos se extraen las funciones de densidad y las funciones de distribución de probabilidad de cada zona sismogénica. 


\section{Planteamiento del problema}

Cumaná, estado Sucre, es una zona sísmicamente activa. Está ubicada en un área próxima a dos sistemas tectónicos, el primero está conformado por el sistema de fallas El Pilar-Casanay, de orientación Este-Oeste, que coexiste con una serie de fallas inversas de rumbo Noreste-Suroeste, y con una serie de fallas de orientación Noroeste, entre ellas las fallas de El Soldado y Los Bajos en el Golfo de Paria, entre el Noreste de Venezuela y Trinidad. El segundo sistema está caracterizado por una porción de litósfera de origen incierto que podría ser parte de la Placa Suramericana o de la placa Atlántica que está subduciendo en sentido Noroeste por debajo de Trinidad y la Península de Paria. (Pérez y Mendoza, 1998). Estos sistemas enmarcan a Cumaná dentro de la zona de deformación producida por la colisión de las placas Caribe, Suramérica y del Atlántico. Esta ubicación está influenciada además por factores que incrementan la probabilidad de daños que un sismo de elevada magnitud causaría a la ciudad, (Rivera, 2017). Desde su primer registro sísmico del 01 de septiembre de 1530 hasta hoy, esta urbe ha sido impactada por muchos sismos, donde destacan varios movimientos mayores que produjeron incalculables daños en bienes materiales, un elevado número de lesionados y pérdida significativa de vidas.

Los sismos son eventos recurrentes que escapan del control humano. Actualmente no es posible señalar con precisión el lugar donde ocurrirá el próximo sismo, tampoco deducir un intervalo de tiempo de ocurrencia lo suficientemente acotado como para tomar acciones a corto o mediano plazo. Las investigaciones en el área, en general convergen en la importancia del estudio y comprensión de los niveles de peligrosidad sísmica con el propósito de diseñar acciones para reducir a largo plazo su impacto sobre la población. En este caso, el propósito de esta investigación es el de construir las funciones de densidad y de distribución de probabilidad de las zonas sismogénicas delineadas para comprender mejor la sismicidad de la ciudad y servir de base a futuros trabajos en el área.

\section{Marco teórico}

El 17 de enero de 1929 y el 9 de julio de 1997 sismos de magnitudes 6,8 y 6,9 Mw respectivamente impactaron la ciudad de Cumaná, (Audemard, 2007) produciendo pérdidas materiales y humanas significativas. Denotando $\left\{X_{i}, i=1,2 \ldots\right\}$ las magnitudes de los sismos que han impactado históricamente la ciudad, se tendría que los sismos de 1929 y 1997 estarían entre los valores máximos de la sucesión $X_{i}$.

Desde el punto de vista estadístico, estos valores están ubicados en la cola de la distribución. Un área de la matemática, denominada Estadística de Valores Extremos (EVE) analiza el comportamiento estocástico de estos valores. Su objeto de estudio son las desviaciones anómalas respecto al valor esperado de una distribución de probabilidad.

\subsection{Distribución de valores extremos}

Consideremos $\left\{X_{1}, \ldots, X_{n}\right\}$ una sucesión de variables aleatorias independientes e idénticamente distribuidas y que tienen la misma función de distribución $\mathrm{F}(\mathrm{x})$. Consideremos además el valor máximo $X_{i} n$ que pueda tomar cada variable $X_{i}$ durante un período de tiempo determinado de observación y reordenemos la sucesión de menor a mayor con base en estos valores

$$
X_{1 n} \leq X_{2 n} \leq \cdots \leq X_{n n}
$$

$X_{1 n}$ y $X_{n n}$ son los valores extremos de esta sucesión. En el caso de los movimientos sísmicos, el interés está en estudiar el comportamiento estadístico de los valores máximos: 


\section{Definición 1}

$M_{n}=X_{n n}=\operatorname{máx}_{i \leq n} X_{i}$ es el máximo de la sucesión $\left\{X_{1}, \cdots, X_{n}\right\}$ donde la distribución de esta variable aleatoria es

$$
F_{M_{n}}(x)=P\left(M_{n} \leq x\right)=\prod_{i=1}^{n} P\left(X_{i} \leq x\right)=F^{n}(x)
$$

Y de la primera derivada, se obtiene su función de densidad

$$
f_{M_{n}}(x)=n[F(x)]^{(n-1)} \cdot f(x)
$$

\section{Definición 2}

$$
\omega(F)=\sup \{x: F(x)<1\}
$$

\section{Definición 3}

Una variable $X$ tiene distribución degenerada (o es degenerada) en un punto c si toma únicamente dicho valor: $X \rightarrow D(c)(c \in R) \Leftrightarrow P(X=c)=1$. En este caso,

$$
F(X)=\left\{\begin{array}{lll}
0 & \text { si } & x<c \\
1 & \text { si } & x \geq c
\end{array}\right.
$$

Si $x<\omega(F)$ entonces $\mathrm{F}(\mathrm{x})<1$ y por tanto $\mathrm{P}\left(\mathrm{M}_{\mathrm{n}} \leq \mathrm{x}\right)=\mathrm{F}^{\mathrm{n}}(\mathrm{x}) \rightarrow 0$ conforme $\mathrm{n} \rightarrow \infty$ además, si $x>\omega(F)$ entonces $F(x)=1$ y por tanto $P\left(M_{n} \leq x\right)=F^{n}(x)=1$.

Esto significa que $\lim _{n \rightarrow \infty} M_{n}=\lim _{n \rightarrow \infty}[F(x)]^{n}=\left\{\begin{array}{lll}1 & \text { si } & F(x)=1 \\ 0 & \text { si } & F(x)<1\end{array}\right.$

así, la distribución del máximo converge a una distribución degenerada. Se tiene, entonces, que hacer una normalización para obtener una distribución límite no degenerada. Para ello se utiliza el Teorema Central del Límite para buscar secuencias de constantes $\left\{a_{n}\right\}$ y $\left\{b_{n}\right\} n \geq 1$, tales que permitan conseguir la transformación lineal

$$
\lim _{n \rightarrow \infty} M_{n}\left(a_{n}(x)+b_{n}\right)=\lim _{n \rightarrow \infty}\left[F\left(a_{n}(x)+b_{n}\right)\right]^{n}=G(x) \forall x ; x \in\left\{X_{1}, \ldots, X_{n}\right\}
$$

con $G(x)$ una distribución no degenerada. Se utiliza para $\left\{a_{n}\right\}$ la media aritmética de la distribución y para $\left\{b_{n}\right\}$ la medida de la varianza con lo que se obtiene que

$$
M_{n}^{*}=\frac{M_{n}-b_{n}}{a_{n}}
$$

converja a una distribución no degenerada cuando $n \rightarrow \infty$. El rango completo de distribuciones límite que podrá seguir la ecuación (5) viene dado por el siguiente teorema:

\section{Teorema 1}

Si existen sucesiones de constantes $\left\{a_{n}>0\right\}$ y $\left\{b_{n}\right\}$ tales que

$$
\mathrm{P}\left(\frac{\mathrm{M}_{\mathrm{n}}-\mathrm{b}_{\mathrm{n}}}{\mathrm{a}_{\mathrm{n}}} \geq \mathrm{x}\right) \rightarrow \mathrm{G}(\mathrm{x}) \text {, cuando } \mathrm{n} \rightarrow \infty
$$

siendo $\mathrm{G}$ una función de distribución no degenerada, entonces $\mathrm{G}$ debe pertenecer a una de las 
siguientes familias con parámetros $\mathrm{a}>0, \mathrm{~b}>0 \mathrm{y} \alpha>0$.

$$
\begin{aligned}
& \text { Gumbel: } G(x)=\exp \left\{-\exp \left[-\left(\frac{x-b}{a}\right)\right]\right\},-\infty<x<\infty \\
& \text { Fréchet: } G(x)= \begin{cases}0, & x \leq b \\
\exp \left\{-\left(\frac{x-b}{a}\right)^{-\alpha}\right\}, & x>b\end{cases} \\
& \text { Weibull: } G(x)= \begin{cases}\exp \left\{-\left[-\left(\frac{x-b}{a}\right)\right]^{\alpha}\right\}, & x<b \\
1, & x \geq b\end{cases}
\end{aligned}
$$

La función $\mathrm{G}(\mathrm{x})$ siempre pertenece a una de estas tres distribuciones sea cual sea la distribución $\mathrm{F}(\mathrm{x})$. Ello implica que la distribución asintótica de los máximos $M_{n}$ se puede estimar sin realizar suposiciones acerca de la distribución original de las observaciones.

Las familias (6), (7) y (8) se pueden combinar en un solo modelo continuo y unificado realizando la reparametrización $\xi=\frac{1}{a}$ y haciendo uso del siguiente teorema denominado Primer Teorema Fundamental de Valores Extremos:

\section{Teorema 2}

Si existen sucesiones de constantes $\left\{a_{n}>0\right\}$ y $\left\{b_{n}\right\}$ tales que

$$
P\left(\frac{M_{n}-b_{n}}{a_{n}} \leq x\right) \rightarrow G(x) \text {, cuando } n \rightarrow \infty
$$

para una distribución $\mathrm{G}$ no degenerada, entonces $\mathrm{G}$ pertenece a la familia de distribuciones de valores extremos,

$$
G(x)=\exp \left\{-\left[1+\xi\left(\frac{x-\mu}{\sigma}\right)\right]^{\frac{-1}{\xi}}\right\}
$$

Esta función está definida en $\left\{x: 1+\xi\left(\frac{x-\mu}{\sigma}\right)>0\right\}$, donde los parámetros de localización, escala y forma satisfacen, $-\infty<\mu<\infty, \sigma>0$ y $-\infty<\xi<\infty$.

Esta familia se denomina distribuciones de valores extremos generalizadas (VEG).

\subsection{Excedencia de umbrales}

\section{Definición 4}

El valor numérico $x_{0}$ perteneciente al rango de valores $\left\{X_{1}, \ldots X_{n}\right\}$ se denomina umbral, si los elementos $x>x_{0}$ son los valores del proceso bajo estudio.

\section{Definición 5}

Sea $x_{0}$ el umbral establecido en la definición 4 . Se denominan valores extremos aquellos valores $x$ ubicados dentro del rango de valores de la sucesión de variables aleatorias $\left\{X_{1}, \ldots X_{n}\right\}$ tales que $x>x_{0}$. 


\section{Definición 6}

Sea $X$ el conjunto de los valores extremos del proceso, se denominan excedencias del umbral $x_{0}$ a los valores $y=x-x_{0}$ para cada elemento $x \in X$.

\section{Definición 7}

Denominaremos $\mathrm{Y}$ al conjunto conformado por las excedencias definidas en 6 .

El comportamiento de $Y$ viene dado por una probabilidad condicional. Consideremos el valor excedentario $e_{i}$ como $e_{i}=x_{0}+y_{i}$ para un elemento $y_{i} \in Y$, según (Villanueva, 2016, pág. 13) esta probabilidad se expresa como

$$
P\left(X>e_{i} / X>x_{0}\right)=\frac{1-F\left(e_{i}\right)}{1-F\left(x_{0}\right)} y_{i} \in Y, y_{i}>0
$$

Si se conociera la distribución F, la distribución de excedencias del umbral sería conocida; como este no es el caso, es necesario el uso de una distribución VEG (familia de distribuciones de valores extremos generalizadas) como aproximación. La distribución de los excesos puede ser determinada exitosamente mediante el siguiente teorema denominado Segundo Teorema Fundamental de Valores Extremos.

\section{Teorema 3}

Sean $X_{1}, X_{2}, \ldots$ una secuencia de variables aleatorias independientes con distribución común $F$, y sea $M_{n}=\operatorname{máx}\left\{X_{1}, \ldots, X_{n}\right\}$. Sea $x$ un término arbitrario $X_{i}$ de esta secuencia y supóngase que $F$ satisface el teorema 2, es decir, $P\left\{M_{n} \leq x\right\} \approx G(x)$, cuando $n \rightarrow \infty$ donde $G(x)=$ $\exp \left\{-\left[1+\xi\left(\frac{x-\mu}{\sigma}\right)\right]^{\frac{-1}{\xi}}\right\}$ para $\mu, \sigma>0$ y $\xi$.

Entonces, para un umbral $x_{0}$ suficientemente grande, la función de distribución de $\left(X-x_{0}\right)$, condicionada a $\mathrm{X}>\mathrm{x}_{0}$, tendrá la forma

$$
H(y)=\left\{\begin{array}{lll}
1-\left(1+\frac{\xi y}{\sigma}\right)^{\frac{-1}{\xi}}, y>0 & \text { si } & \xi \neq 0 \\
1-\exp \left(\frac{-y}{\sigma}\right), y>0 & \text { si } & \xi=0
\end{array}\right.
$$

estando definida en $\left\{y: y>0 y\left(1+\frac{\xi y}{\sigma}\right)^{\frac{1}{\xi}}>0\right\}$, donde

$$
\sigma=\sigma+\xi\left(x_{0}-\mu\right)
$$

Llamase a este grupo de distribuciones, familia de distribuciones de Pareto generalizada (DPG) y está caracterizada por tres parámetros, el parámetro de localización $\mu$, el parámetro de escala $\sigma$ y el de forma $-\infty<\xi<+\infty$. Si $\xi<0$, la distribución de los excesos tiene como límite superior a $\mu-\frac{\sigma}{\xi}$. Si $\xi \geq 0$, no tiene límite superior. Para $\xi>0$ se tiene la distribución de Pareto ordinaria. En el caso de que $\xi=0$, se corresponde con una distribución exponencial de parámetro $\frac{1}{\sigma}$.

\section{3. Área de estudio}

Cumaná, está ubicada espacialmente en los $10^{\circ} 27^{\prime} 14^{\prime \prime}$ de latitud norte $\left(10,45^{\circ}\right.$ en grados decimales) y $64^{\circ} 10^{\prime} 57^{\prime \prime}$ de longitud oeste $\left(-64,18^{\circ}\right)$ y tiene una altitud promedio de 4 metros sobre el nivel del mar. Para esta investigación se toma como área de estudio un radio de $300 \mathrm{Km}$. alrededor de la ciudad, tomando como valores de referencia $1^{\circ}$ latitud $=111,1 \mathrm{Km}$. y $1^{\circ}$ longitud $=111,32 \mathrm{Km}$., el área de estudio se circunscribe entonces a una región ubicada entre $\operatorname{los} 7^{\circ}$ y $13^{\circ}$ de latitud y entre $\operatorname{los}-61^{\circ}$ y $-67^{\circ}$ de longitud. 


\subsection{Catálogo sísmico}

Se utilizó un catálogo sísmico tomado de la investigación de (Rivera, 2017); cuya información sismológica instrumental se obtuvo de los boletines del Centro de Sismología de la UDO (CSUDO) desde 1995 hasta 2012, el Boletín Sismológico Nacional de FUNVISIS desde 1910 hasta 2011, el catálogo telesísmico del National Earthquake Information Center (NEIC) y del United States Geological Survey (USGS) para el período comprendido entre 1976 y enero de 2016. El catálogo incluye los sismos históricos, no instrumentales recopilados por diversos autores. Se entiende por sismos no instrumentales aquellos sismos cuya magnitud se deduce de los relatos y crónicas de la época cuando ocurrió debido a la inexistencia de instrumentos específicos para esta medición. Al catálogo se le realizó la depuración de los sismos que carecían de localización y fecha, también los eventos duplicados; la homogenización de magnitudes, el filtrado de réplicas y el análisis de completitud; además se tomó como umbral de referencia la magnitud 4,0 Mw por considerar que los sismos de magnitud inferior a esta no contribuyen de manera significativa en el cálculo de la peligrosidad. Finalmente, se seleccionaron los sismos con epicentros ocurridos entre las coordenadas $-61^{\circ}$ y $-67^{\circ}$ de longitud y entre los $7^{\circ}$ y los $13^{\circ}$ de latitud. Se obtuvo un total de 822 sismos instrumentales y 7 sismos históricos. Posteriormente, se subdividió el conjunto de datos considerando la profundidad del sitio exacto bajo el terreno donde se originó el sismo (hipocentro): 307 sismos superficiales $(0-30 \mathrm{~km}) ; 226$ sismos de profundidad intermedia, (30 - $70 \mathrm{~km}$ ); 296 sismos profundos, (Más de $70 \mathrm{~km}$ ).

\section{Análisis de los datos}

Para cada zona sismogénica se toman las magnitudes de los sismos $(\mathrm{m})$ y el número de sismos que superan esas magnitudes $(N)$. Al parámetro $N$ se le calcula su logaritmo decimal $(\log (N))$. Dado que la ley de Gutenberg-Ritcher establece que para un período de tiempo determinado y en una cierta zona

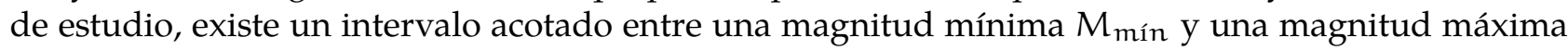

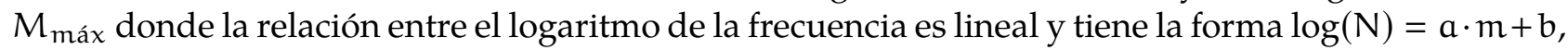
se procede a realizar un análisis de regresión lineal utilizando el método de mínimos cuadrados para obtener los parámetros a y b para cada zona sismogénica. Estos parámetros se utilizan para calcular los valores $\alpha=a \cdot \ln (10), \beta=b \cdot \ln (10)$ para cada zona sismogénica.

La ecuación de Gutemberg-Ritcher dada anteriormente, puede transformarse mediante un procedimiento matemático para llegar a la expresión

$$
N(m)=e^{\alpha-\beta M_{\text {mín }}} \cdot e^{-\beta\left(m-M_{\text {mín }}\right)}
$$

Haciendo $N_{M_{\text {mín }}}=e^{\alpha-\beta M_{\text {mín }}}$

tendremos $N(m)=N_{M_{\text {mín }}} \cdot e^{-\beta\left(m-M_{\text {mín }}\right)}$

Desde aquí se obtiene la función de distribución de probabilidad para cada zona sismogénica de la siguiente manera

$$
\begin{aligned}
& F(m)=P\left[m<M \mid m>M_{\text {mín }}\right]=\frac{N_{M_{\text {min }}}-N(m)}{N_{M_{\text {mín }}}} \\
& F(m)=\frac{N_{M_{\text {min }}}-N_{M_{\text {min }}} \cdot e^{-\beta\left(m-M_{\text {mín }}\right)}}{N_{M_{\text {min }}}}=1-e^{-\beta\left(m-M_{\text {mín }}\right)}
\end{aligned}
$$

La función de densidad para cada zona sismogénica, se deduce diferenciando $F(m)$

$$
f(m)=\frac{d(F(m))}{d m}=\beta e^{-\beta\left(m-M_{\text {mín }}\right)}
$$




\subsection{Sismicidad entre 0 y $30 \mathrm{~km}$ de profundidad}

Del catálogo sísmico tomado de la investigación de (Rivera, 2017), entre 0 y $30 \mathrm{~km}$ de profundidad se ubican 307 focos de sismos, de los cuales, 195 están en el intervalo 4, $0 \leq \mathrm{Mw}<5,0$; 92 en el intervalo $5,0 \leq M w<6,0$ y 20 son de magnitud $M w \geq 6,0$.

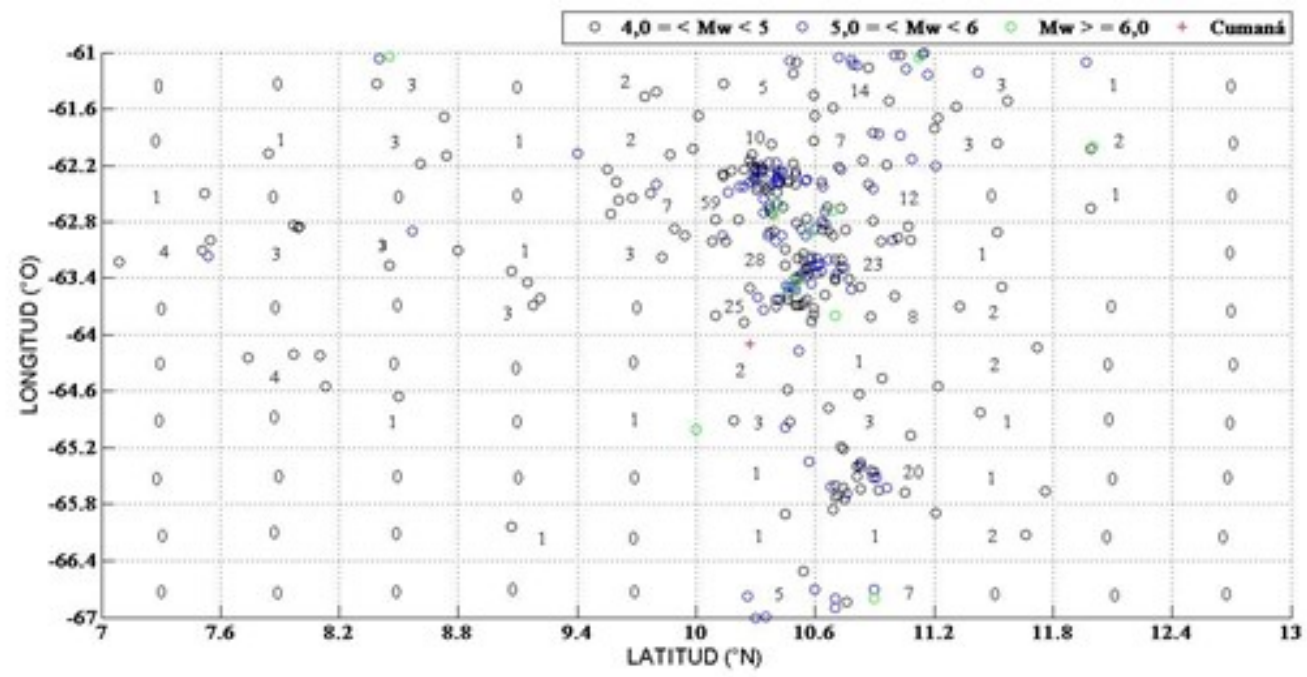

Figura 1: Malla de sismos con hipocentros entre 0 y 30 Kms. Elaboración propia.

A partir de las zonas de mayor densidad sísmica y tomando en cuenta los sismos históricos, la geología de la zona y la magnitud de los sismos se determinan las zonas sismogénicas I, II y III.

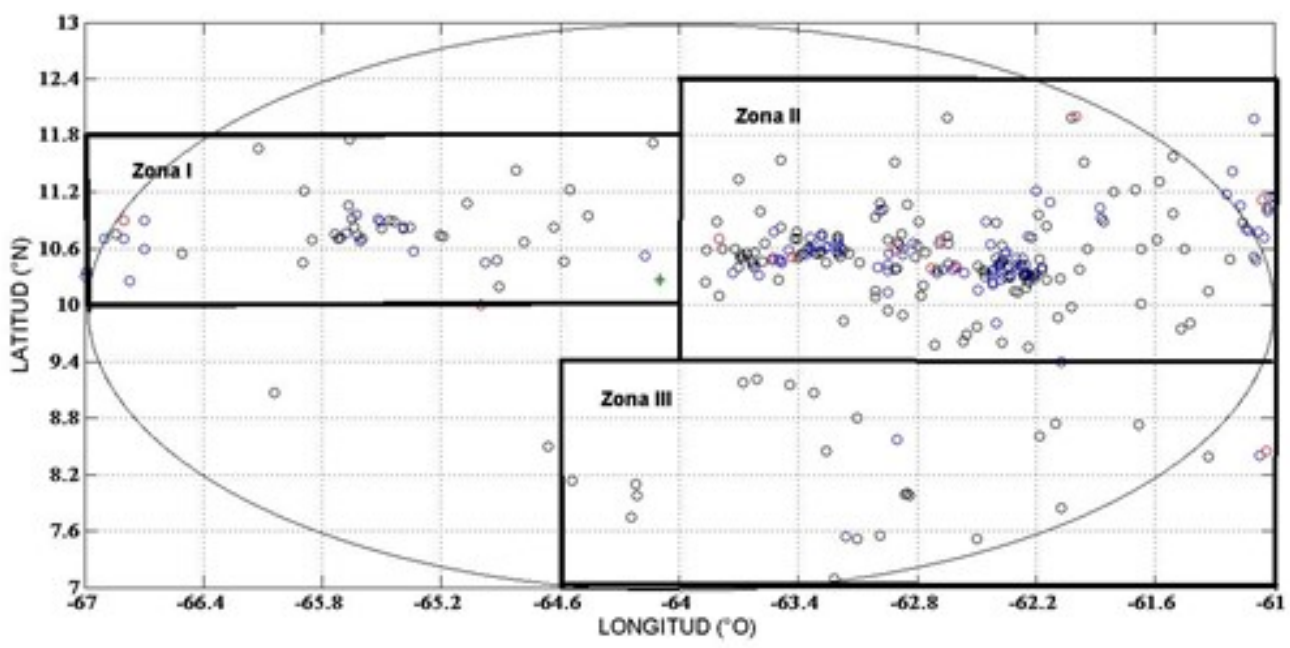

Figura 2: Zonas sismogénicas I, II y III. Elaboración propia.

La zona I se ubicó hacia el oeste de la ciudad con 53 sismos instrumentales y 5 sismos históricos. La zona II hacia el este con 219 sismos instrumentales y 1 sismo histórico. La zona III se ubicó al sur y sureste con 26 sismos instrumentales y 1 sismo histórico. Un remanente de 2 sismos no se ubicaron en zona sismogénica alguna. A partir de las excedencias obtenidas para los sismos ubicados en cada zona se construyen las curvas de densidad y de distribución de probabilidad. 
Zona sismogénica I:

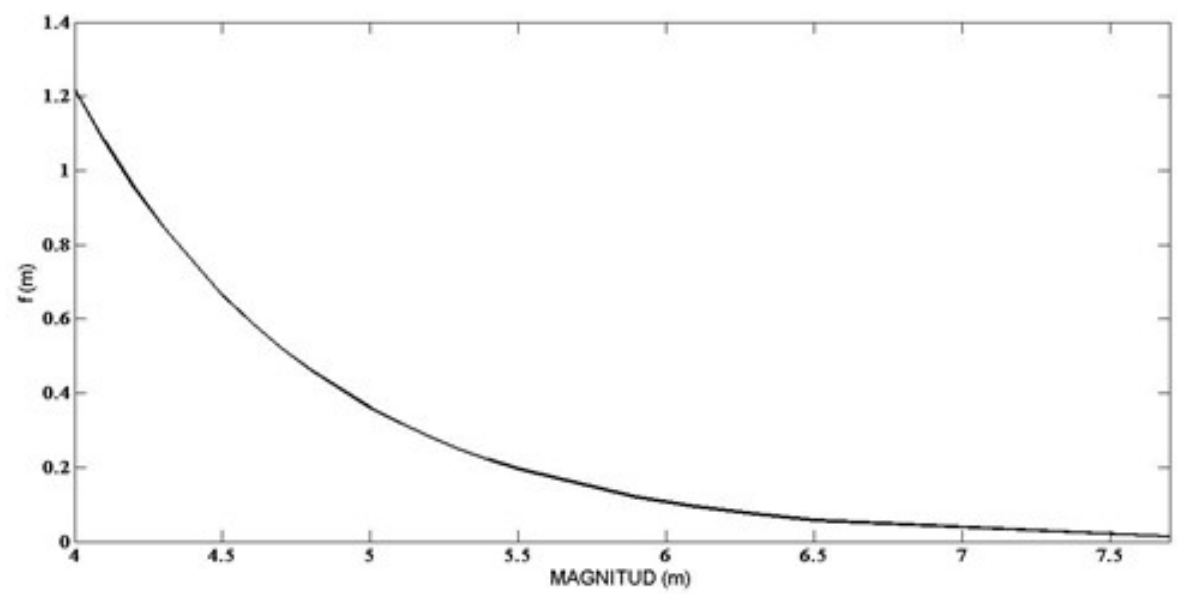

Figura 3: Función de densidad de la zona I. Elaboración propia.

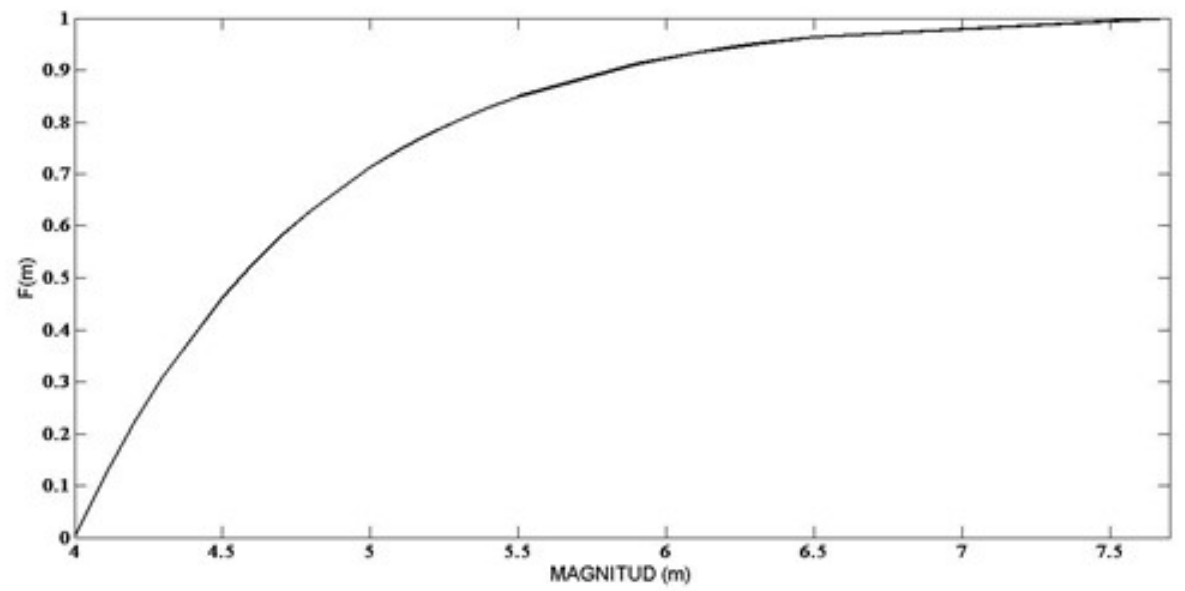

Figura 4: Función de distribución de probabilidad de la zona I. Elaboración propia.

Zona sismogénica II:

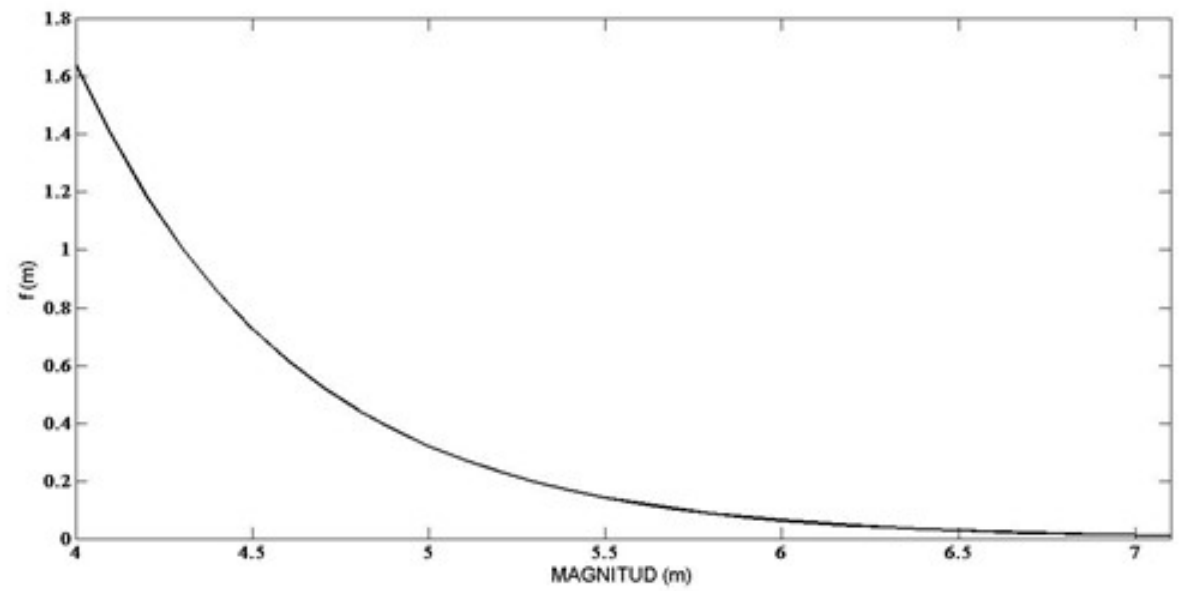

Figura 5: Función de densidad de la zona II. Elaboración propia. 


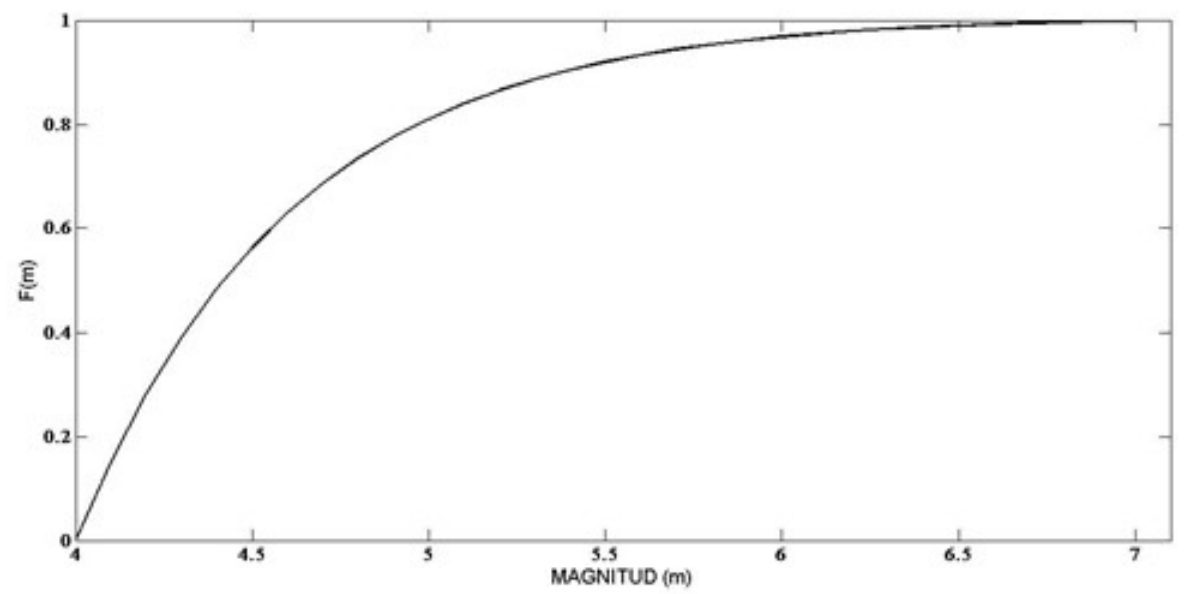

Figura 6: Función de distribución de probabilidad de la zona II. Elaboración propia.

\section{Zona sismogénica III:}

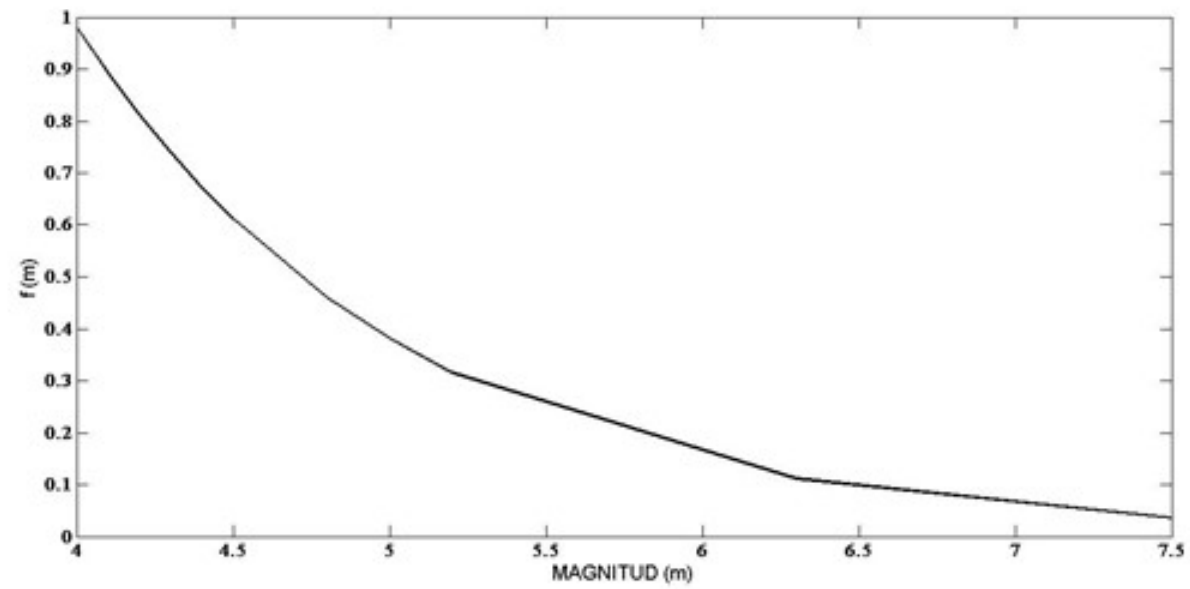

Figura 7: Función de densidad de la zona III. Elaboración propia.

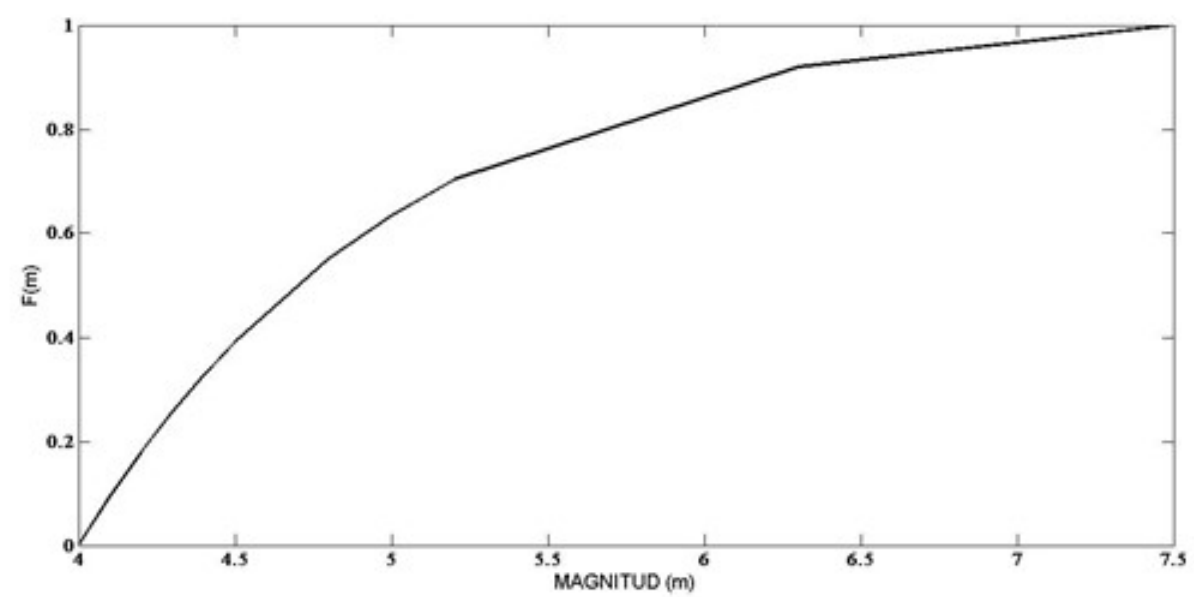

Figura 8: Función de distribución de probabilidad de la zona III. Elaboración propia. 


\subsection{Sismicidad entre 30 y $70 \mathrm{~km}$ de profundidad}

Del catálogo sísmico tomado de la investigación de (Rivera, 2017), entre 30 y $70 \mathrm{~km}$ de profundidad se ubican 226 focos de sismos, 132 están en el intervalo 4, $0 \leq M w<5,0 ; 82$ en 5,0 $\leq M w<6,0$ y 13 son de magnitud $M w \geq 6,0$.

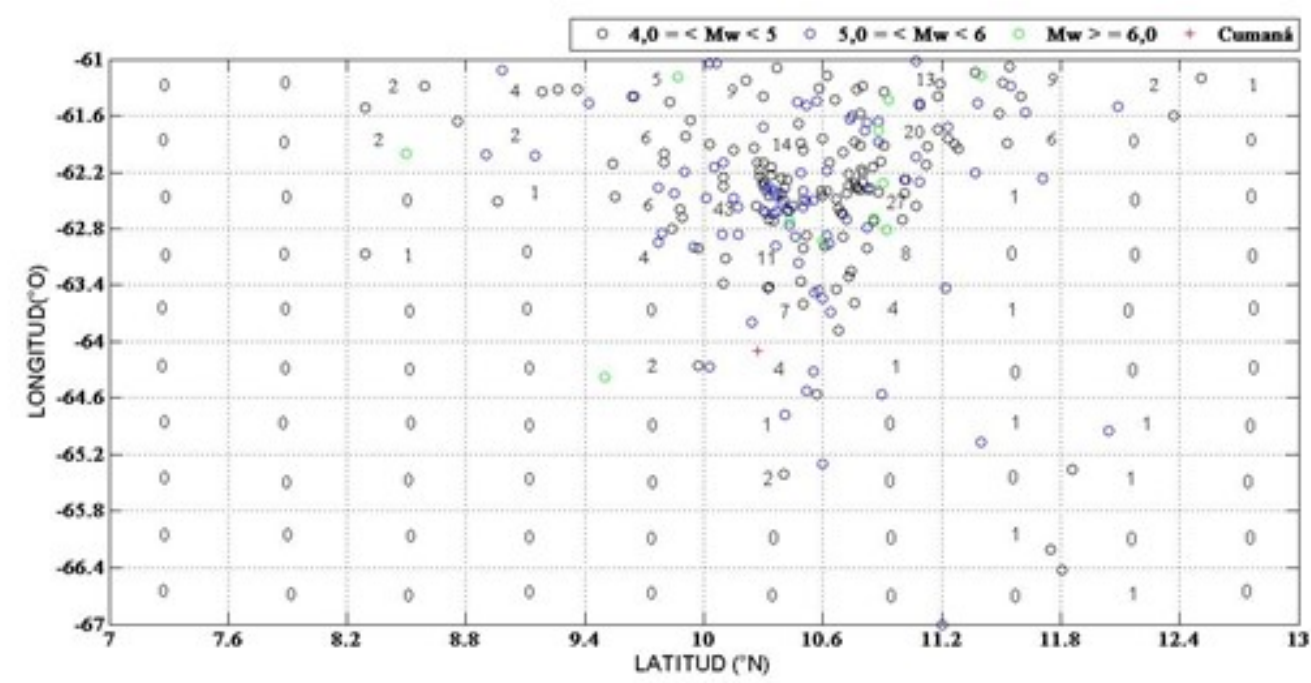

Figura 9: Malla de sismos con hipocentros entre 30 y $70 \mathrm{Km}$. Elaboración propia.

A partir de las zonas de mayor densidad sísmica, la geología de la zona y la magnitud de los sismos se determinan las zonas sismogénicas IV y V.

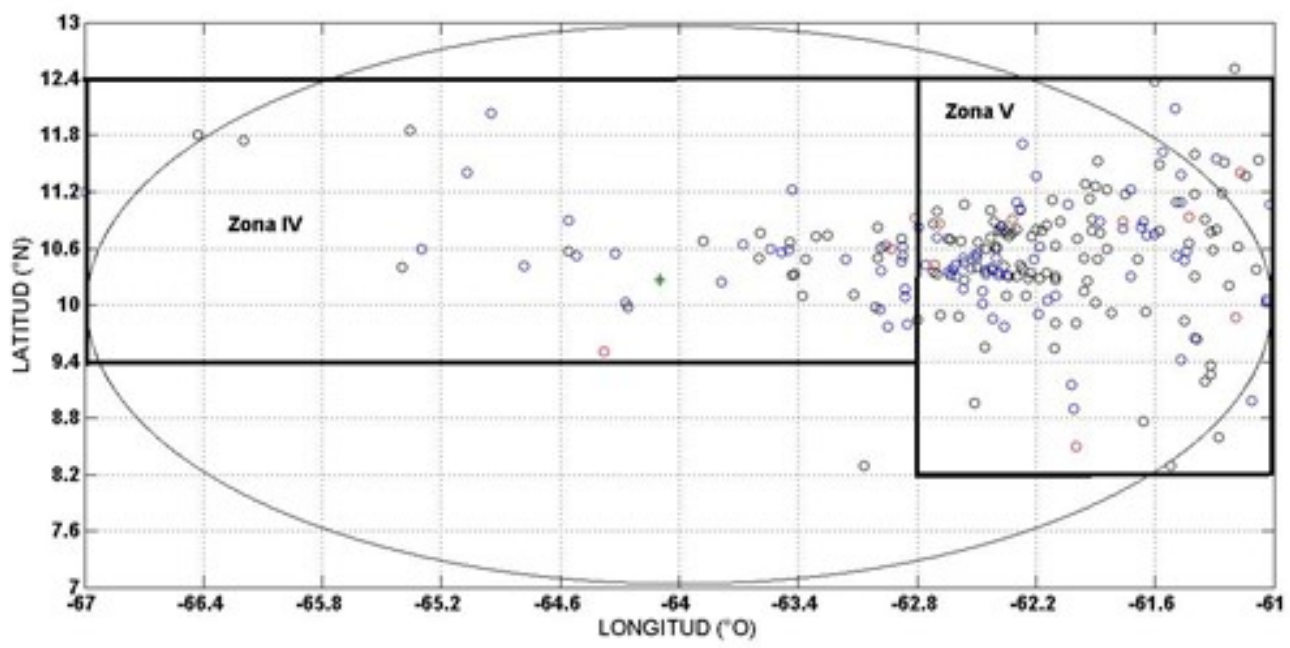

Figura 10: Zonas sismogénicas IV y V. Elaboración propia.

La zona IV se ubicó al oeste y en los alrededores de la ciudad, allí hay reportes de 50 sismos instrumentales y 1 sismo histórico con un claro predominio de sismos de baja magnitud. La zona $V$ asociada al arco de las Antillas menores está situada muy al este de la ciudad y presenta gran densidad sísmica (172 sismos instrumentales) y predominio de sismos con magnitudes entre 4,5 y $5.3 \mathrm{Mw}$. Un remanente de 2 sismos no se ubicaron en zona sismogénica alguna. 
Zona sismogénica IV:

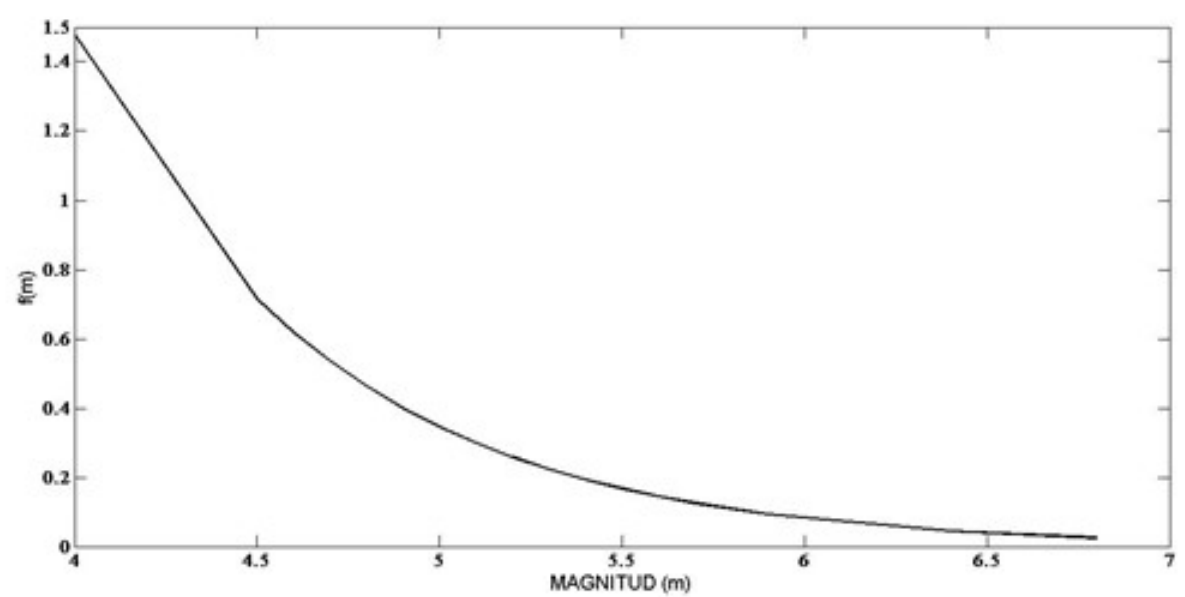

Figura 11: Función de densidad de la zona IV. Elaboración propia.

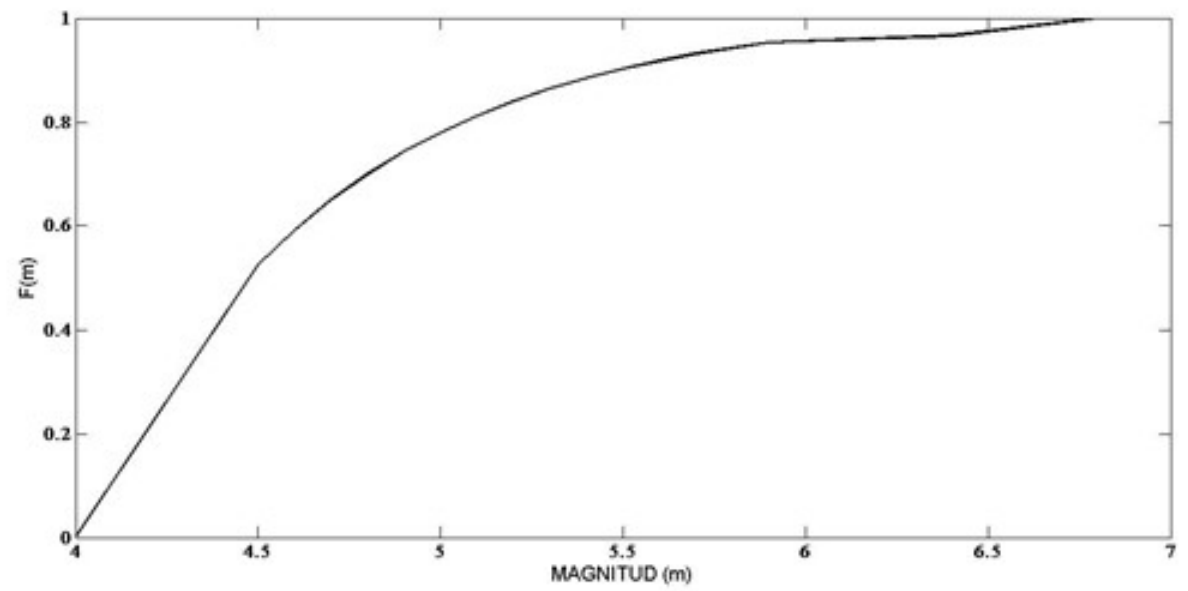

Figura 12: Función de distribución de probabilidad de la zona IV. Elaboración propia.

Zona sismogénica V:

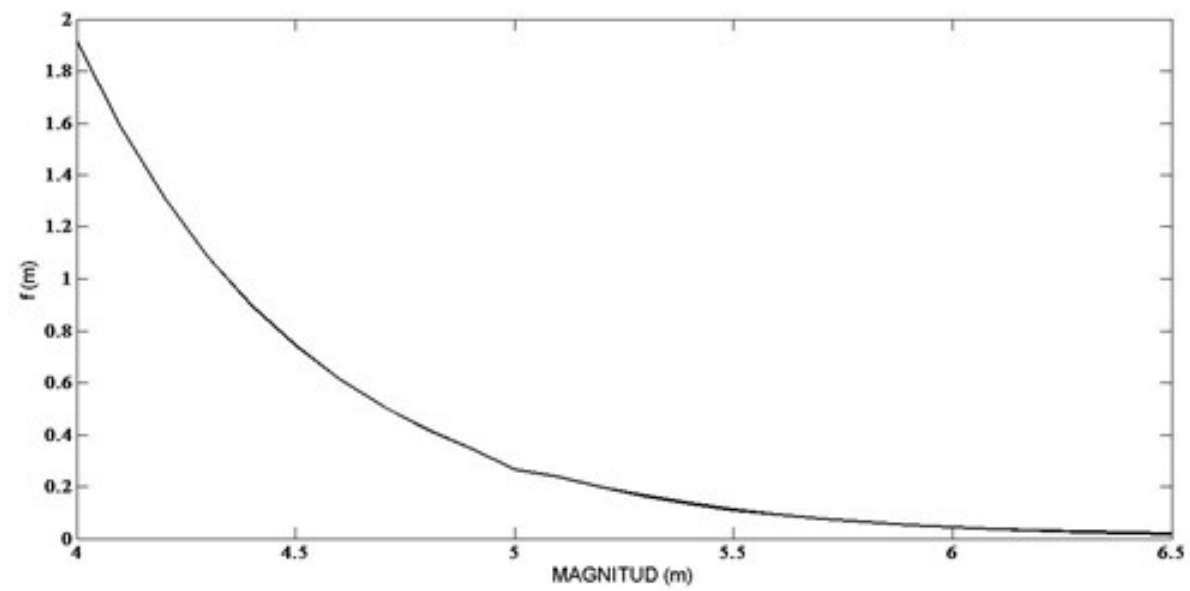

Figura 13: Función de densidad de la zona V. Elaboración propia. 


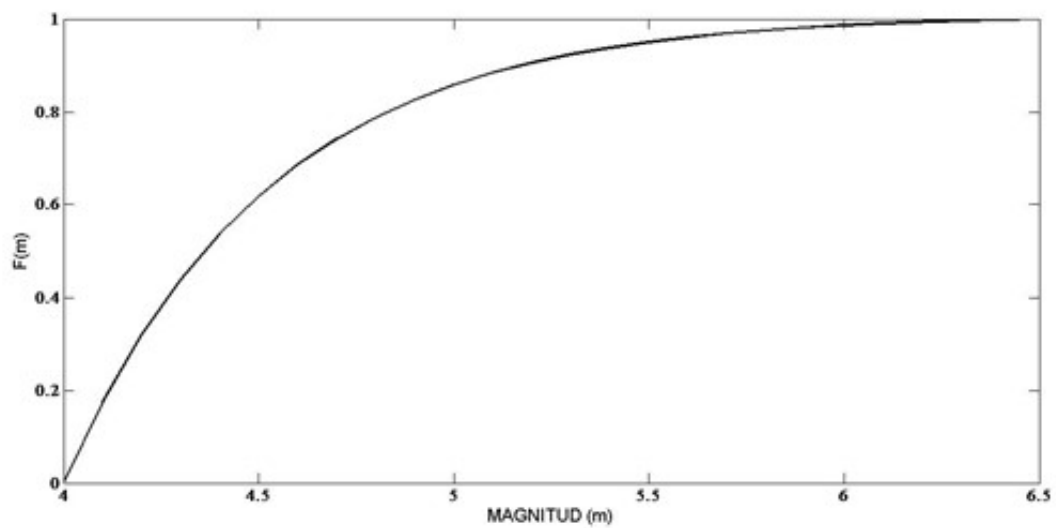

Figura 14: Función de distribución de probabilidad de la zona V. Elaboración propia.

\subsection{Sismicidad a más de $70 \mathrm{~km}$ de profundidad}

Del catálogo sísmico tomado de la investigación de (Rivera, 2017), a más de $70 \mathrm{~km}$ de profundidad se ubican 296 focos de sismos, de ellos 176 están en el intervalo 4, $0 \leq M w<5,0 ; 109$ en el intervalo $5,0 \leq M w<6,0$ y 11 son de magnitud $M w \geq 6,0$.

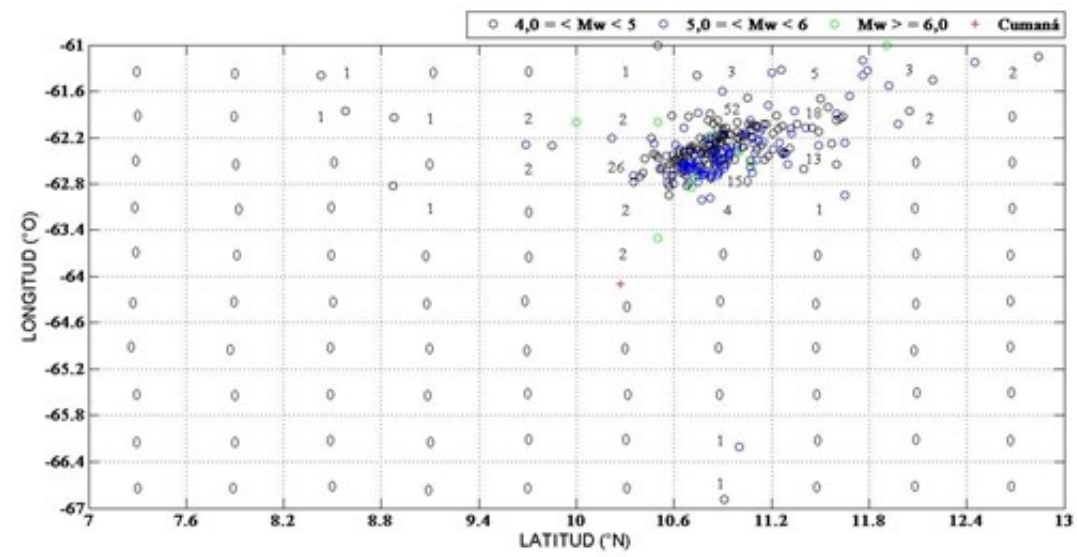

Figura 15: Malla de sismos con hipocentros a más de 70 km. Elaboración propia.

A partir de las zonas de mayor densidad sísmica y tomando en cuenta la geología de la zona y la magnitud de los sismos se determina la zona VI.

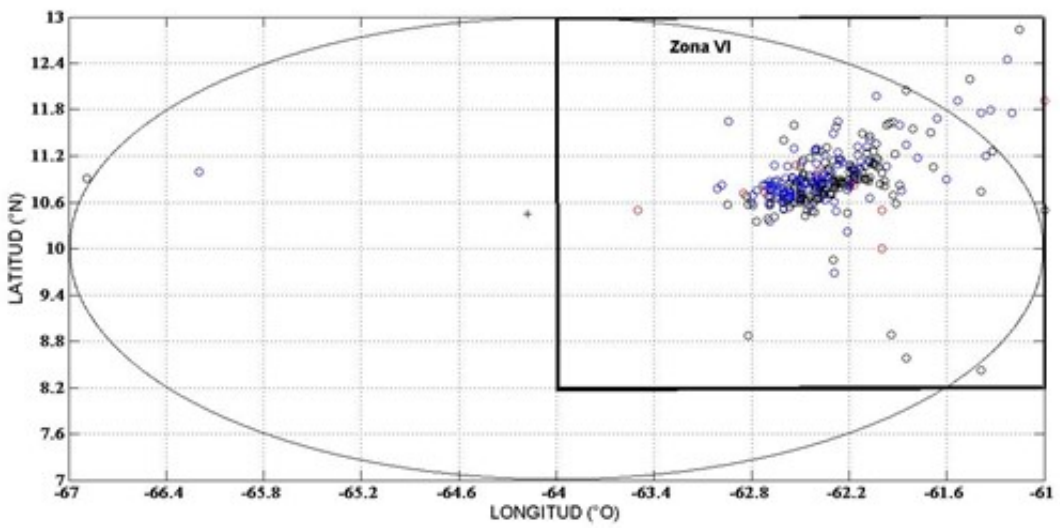

Figura 16: Zona sismogénica VI. Elaboración propia. 
En esta gráfica se visualiza una zona sismogénica (zona VI) que abarca la casi totalidad de los sismos reportados. Un remanente de 2 sismos quedan fuera de la delimitación de la zona sismogénica.

Zona sismogénica VI:

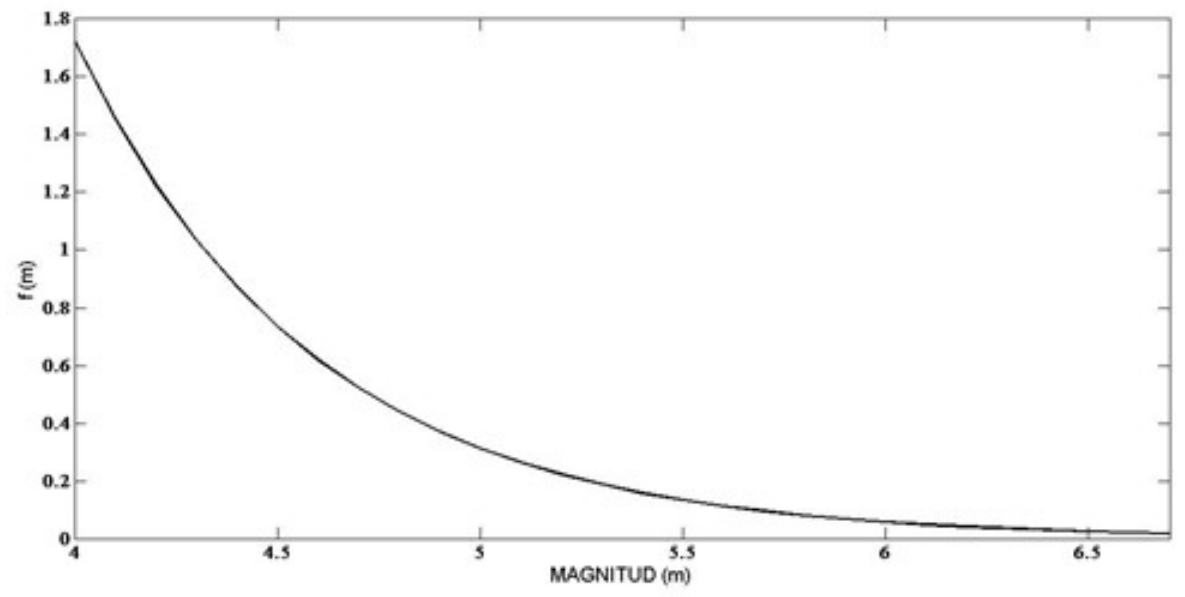

Figura 17: Función de densidad de la zona VI. Elaboración propia.

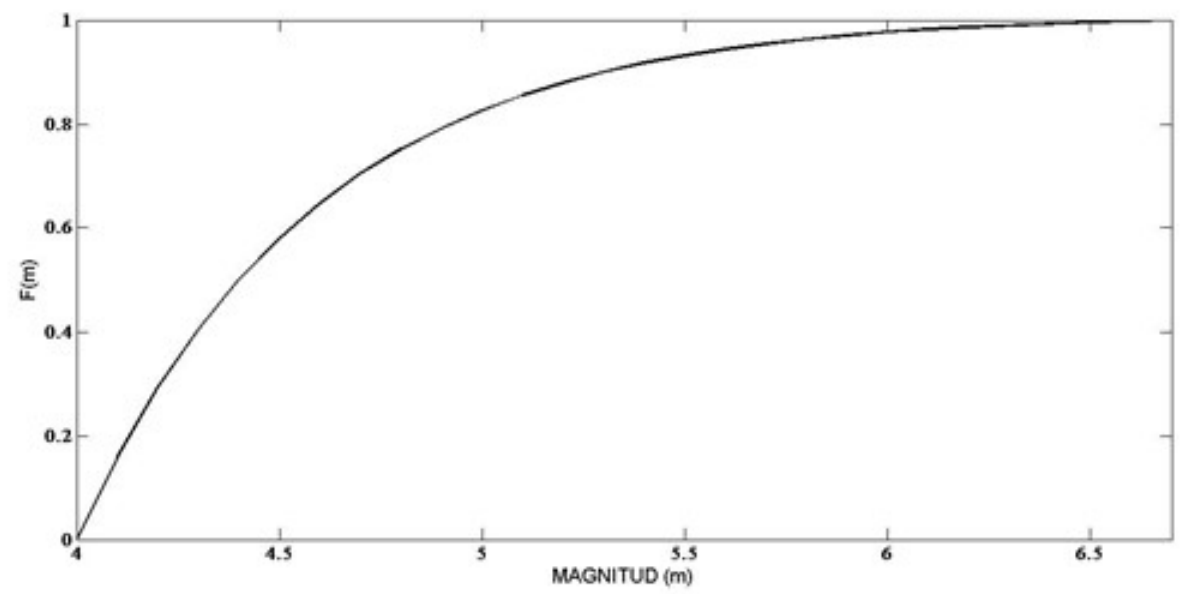

Figura 18: Función de distribución de probabilidad de la zona VI. Elaboración propia.

\section{Conclusiones}

La mayor densidad sísmica para los sismos con hipocentros ubicados en profundidad entre 0 y $30 \mathrm{~km}$ y los de 30 a $70 \mathrm{~km}$ se ubica en una franja entre $l o s 10^{\circ}$ y $11,8^{\circ}$ de latitud que se corresponde con el trazo principal de la falla El Pilar. Para los sismos con más de $70 \mathrm{~km}$ de profundidad la mayor densidad sísmica se corresponde a un área ubicada entre las mismas latitudes anteriores, pero circunscritas a un sector entre los $-62^{\circ}$ y los $-61^{\circ}$ de longitud que es el área de subducción ubicada hacia el noreste de la ciudad en la zona de convergencia de las placas del Caribe, Suramérica y del Atlántico.

Las funciones de distribución de probabilidad obtenidas para cada zona, tienen la forma de una distribución de Pareto tal y como lo plantea el segundo teorema fundamental de valores extremos.

En el lado derecho, cada una de las funciones de densidad obtenidas se aproximaron de distintas 
maneras a la asíntota, notándose una cola más pesada en la figura 5 correspondiente a la zona II y la 13 correspondiente a la zona V. La caída menos pronunciada se observa en la figura 7 , que pertenece a la zona III. Las funciones de distribución de probabilidad construidas se aproximan a 1 también de manera similar, siendo las colas más pesadas las de la figura 4 que atañe a la zona I y la 14 que corresponde a la zona V.

Sería interesante para futuras investigaciones la visualización y estudio de otros parámetros estadísticos, que no son objetos de estudio actualmente.

\section{Referencias}

[1] Audemard, F. (2007). "Revised seismic history of El Pilar Fault, Northeastern Venezuela, after Cariaco 1997 Earthquake and from recent preliminary paleoseismic results". Journal of Seismology, 11 (3): 311-326.

[2] Benito, B. y Jiménez, M. (1999). “Peligrosidad Sísmica”. Física de la Tierra, 11: 13-47.

[3] Mayordomo, J. (2005). Caracterización y análisis de la peligrosidad sísmica en el sureste de España. Tesis Doctoral. Universidad Complutense de Madrid.

[4] Pérez, O. y Mendoza, J. (1998). “Sismicidad y tectónica en Venezuela y áreas vecinas". Física de la Tierra, 10: 87-110.

[5] Rivera, Y. (2017). Evaluación del riesgo sísmico en Cumaná, Estado Sucre. Tesis de Maestría. Universidad de Oriente, Cumaná.

[6] Serra, I. (2013). Modelos estadísticos para valores extremos y aplicaciones. Universidad Autónoma de Barcelona. España.

[7] Villanueva, A. (2016). Análisis de Valores Extremos. Modelización espacial. Universidad de Granada. España. 